\title{
Intracranial MR-guided laser-induced thermal therapy: single-center experience with the Visualase thermal therapy system
}

\author{
Purvee Patel, BA,,$^{1,2}$ Nitesh V. Patel, MD, ${ }^{2,3}$ and Shabbar F. Danish, MD ${ }^{1}$ \\ ${ }^{1}$ Cancer Institute of New Jersey, Rutgers University; ${ }^{2}$ Department of Neurological Surgery, Rutgers-Robert Wood Johnson \\ Medical School, New Brunswick; and ${ }^{3}$ Department of Neurological Surgery, Rutgers-New Jersey Medical School, Newark, \\ New Jersey
}

\begin{abstract}
OBJECTIVE MR-guided laser-induced thermal therapy (MRgLITT) can be used to treat intracranial tumors, epilepsy, and chronic pain syndromes. Here, the authors report their single-center experience with 102 patients, the largest series to date in which the Visualase thermal therapy system was used.
\end{abstract}
METHODS A retrospective analysis of all patients who underwent MRgLITT between 2010 and 2014 was performed. Pathologies included glioma, recurrent metastasis, radiation necrosis, chronic pain, and epilepsy. Laser catheters were placed stereotactically, and ablation was performed in the MRI suite. Demographics, operative parameters, length of hospital stay, and complications were recorded. Thirty-day readmission rates were calculated by using the standard method according to America's Health Insurance Plans Center for Policy and Research guidelines.

RESULTS A total of 133 lasers were placed in 102 patients who required intervention for intracranial tumors (87 patients), chronic pain syndrome (cingulotomy, 5 patients), or epilepsy (10 patients). The procedure was completed in $98 \%$ $(100)$ of these patients. Ninety-two patients $(90.2 \%)$ had undergone previous treatment for their intracranial tumors. The average $( \pm$ SD) total procedural time was $170.5 \pm 34.4$ minutes, and the mean laser-on time was $8.7 \pm 6.8$ minutes. The average intensive care unit (ICU) and hospital stays were 1.8 and 3.6 days, respectively, and the median length of stay for both the ICU and the hospital was 1 day. By postoperative Day $1,54 \%$ of the patients $(n=55)$ were neurologically stable for discharge. There were 27 cases of morbidity, including new-onset neurological deficits, and 2 perioperative deaths. Fourteen patients (13.7\%) developed new deficits after the MRgLITT procedure, and of those 14 patients, $64.3 \%$ $(n=9)$ had complete resolution of deficits within 1 month, $7.1 \%(n=1)$ had partial resolution of symptoms within 1 month, $14.3 \%(n=2)$ had not had resolution of symptoms at the most recent follow-up, and $14.3 \%(n=2)$ died without resolution of symptoms. The 30 -day readmission rate was $5.6 \%$

CONCLUSIONS MRgLITT, although minimally invasive, must be used with caution. Thermal damage to critical and eloquent structures can occur despite MRI guidance. Once the learning curve is overcome, the overall procedural complication rate is low, and most patients can be discharged within 24 hours, with a relatively low readmission rate. In cases in which they occurred, most neurological deficits were temporary. The therapeutic role of MRgLITT in various intracranial diseases will require larger and more rigorous studies.

http://thejns.org/doi/abs/10.3171/2015.7.JNS15244

KEY WORDS MRI-guided laser ablation; Visualase; safety profile; laser interstitial thermal therapy; surgical technique

$\mathrm{W}$ ITH the widespread use of image guidance for neurosurgical procedures, minimally invasive methodologies have flourished. Advances in laser construction and quantitative thermal imaging based on proton resonance frequency shift have allowed this technology to be reexamined for intracranial pathologies. ${ }^{11}$ Furthermore, the addition of thermal damage estimates enables real-time estimation of thermally induced damage, which potentially allows for safer treatments. ${ }^{4}$
The Visualase thermal therapy system (Medtronic, Inc) combines a therapeutic laser applicator with an imageprocessing workstation to provide a system for real-time MR-guided laser thermal therapy. ${ }^{14,15}$ A $15-\mathrm{W}, 980$-nm diode laser supplies energy to a 400- $u$ m core fiberoptic applicator with a light-diffusing tip constructed from a silicone elastomer with embedded scattering particles. This applicator produces a roughly cylindrical to ellipsoid light distribution in the tissue along the axis of the diffusing ele-

ABBREVIATIONS ICU = intensive care unit; MRgLITT = MR-guided laser-induced thermal therapy.

SUBMITTED February 2, 2015. ACCEPTED July 28, 2015.

INCLUDE WHEN CITING Published online January 1, 2016; DOI: 10.3171/2015.7.JNS15244. 
ment. The laser-diffusing fiber itself is used inside a coaxial cooling catheter connected to a peristaltic roller pump that circulates sterile saline to cool the fiber and adjacent tissue. This system has been used in the treatment of a variety of intracranial tumors, epilepsy, and chronic pain syndromes. . $^{26-8,16,18,21-23}$ Furthermore, outside of neurosurgery, this procedure has been used to treat pulmonary, hepatic, ${ }^{32-34}$ bone, breast, abdominal, and prostate masses. ${ }^{1,20,28,29,32-34}$ Once therapy begins, the thermal energy causes intracellular damage that leads to cell death. The MR-guided laser-induced thermal therapy (MRgLITT) software allows for the setting of temperature boundaries and for evaluation of the extent of ablation. As the target is ablated, real-time tissue temperature changes and ablation are seen on the MR image.

To date, the literature has been limited to reports of small series of patients that mostly documented clinical outcomes from tumor or epilepsy treatments. ${ }^{2-4,6,9,10} \mathrm{Un}$ derstanding the safety profile for MRgLITT is critical, because previous reports have mentioned the risk of postablation edema, hemorrhage, and hyperthermia-induced injury to nearby eloquent structures..$^{19,26,35} \mathrm{We}$ report here our experience using the Visualase thermal therapy system in more than 100 patients with a variety of intracranial pathologies. We describe the procedural safety profile, perioperative morbidity and mortality rates, and the occurrence of neurological deficits across the treated pathologies. To our knowledge, this is the largest experience to date with the Visualase thermal therapy system.

\section{Methods \\ Patient Selection}

Patients were selected to undergo this procedure as part of the routine clinical algorithm at our institution depending on their pathology and corresponding treatment course. The following 4 categories of indications were identified: 1) high-grade primary tumors, 2 ) secondary tumors, 3) epilepsy, and 4) chronic pain. Typically, for tumor cases, MRgLITT was performed after resection or other adjuvant options (radiotherapy and chemotherapy) were exhausted or when the tumor was deemed inoperable using traditional resection. In the case of epilepsy, only those patients with clearly localized seizure foci, as with medial temporal lobe sclerosis, were offered MRgLITT. Bilateral anterior cingulotomy was performed using this system for refractory pain syndromes. Overall, patients were offered all available options in addition to MRgLITT. All patients were enrolled in an institutional review board-approved research protocol.

A total of 102 patients were treated between July 2010 and July 2014 at Robert Wood Johnson University Hospital. Table 1 summarizes patient demographic information. All procedures were performed directly or overseen by the senior author (S.F.D.). The average patient age was $53 \pm 20.3$ years (range 1-85 years). There was an even distribution of males $(n=51)$ and females $(n=51)$. A majority of the patients underwent the procedure for a primary brain tumor (n $=50)$. The pathologies included glioma $(\mathrm{n}=8)$, glioblastoma multiforme $(n=24)$, ependymoma $(n=3)$, meningioma $(\mathrm{n}=2)$, primitive neuroectodermal tumor $(\mathrm{n}=3)$, heman-
TABLE 1. Demographic and diagnostic data for the 102 patients

\begin{tabular}{cc}
\hline \multicolumn{1}{c}{ Demographic } & No. of Patients $(\%)$ \\
\hline Age $(\mathrm{yrs})$ & \\
\hline Mean & 53 \\
\hline Range & $1.25-85$ \\
\hline Sex & $51(50)$ \\
\hline Female & $51(50)$ \\
\hline Male & \\
\hline Diagnosis & $50(49.0)$ \\
\hline Primary brain tumor/glioma & $37(36.3)$ \\
\hline RecMets/RadNec & $5(4.9)$ \\
\hline Pain & $10(9.8)$ \\
\hline Epilepsy & $102(100)$ \\
\hline Total
\end{tabular}

RecMets $/$ RadNec $=$ recurrent metastatic tumors/radiation necrosis .

gioblastoma ( $\mathrm{n}=2$ ), cavernoma (2), and chordoma, pilocytic astrocytoma, teratoma, pineal tumor, CNS lymphoma, and ganglioglioma $(\mathrm{n}=1$ each). The second most common diagnosis that necessitated the procedure was recurrent metastatic tumor/radiation necrosis $(\mathrm{n}=37)$. Other indications for MRgLITT included cingulotomy for chronic refractory pain syndrome $(\mathrm{n}=5)$ and epilepsy $(\mathrm{n}=10)$.

\section{Operative Procedure}

We previously detailed the operative procedure and setup. ${ }^{10}$ Preoperatively, CT and MR imaging sequences are acquired and used for stereotactic planning through the Medtronic StealthStation S7 (Medtronic, Inc).

During the preparation, the patient is placed into a Mayfield clamp and positioned. CT and MRI images are fused, and stereotactic registration is performed using the StealthStation S7 system. All procedures are performed using frameless stereotaxy. Once the trajectory and targeting plan are confirmed, the "probe's eye" view feature is then used to ensure that the planned trajectory does not violate any blood vessels or cross sulci. The precision aiming device is connected to the stealth arm connector, and the stealth pointer is used to confirm the trajectory. Next, a stab incision is made with a No. 15 blade, and a reducing cannula is placed in the precision aiming device to accommodate a $3.2-\mathrm{mm}$ power drill. A bur hole is created, and a spinal needle is used to puncture the dura. A $2.2-\mathrm{mm}$ reducing cannula is inserted, and a bone anchor is placed, which secures the laser catheter.

After the patient is transported for MRI and the laser catheter(s) are connected to the Visualase system, we typically deliver a test dose at $4 \mathrm{~W}$ for $10-15$ seconds. High temperature limits $\left(90^{\circ} \mathrm{C}\right)$ are set near the tip of the applicator to avoid the production of steam. Low temperature limits $\left(50^{\circ} \mathrm{C}\right)$ are set at the borders of the target area or near critical structures to avoid unintended thermal damage. Furthermore, real-time thermal damage is estimated by the system based on MRI pixel shift of the target tissue in response to thermal damage. Once ablation is complete, a single suture is usually used to close the scalp after removal of the laser catheter. 


\section{Data Collection and Analysis}

Patient demographic data and operative variables were collected retrospectively. Operative parameters, including preparation time, time in the operating room, time for transfer for MRI, time spent in the MRI suite, time for laser ablation, and total operative time, were recorded prospectively. New neurological deficits, such as weakness, gait instability, and cognitive decline, were ascertained from hospital and follow-up records. Perioperative complications, including inaccurate laser placement, insertional or ablation-induced hemorrhage, refractory postprocedure edema, infection, or death secondary to the procedure, were also recorded. Postprocedural refractory edema was defined as that which was persistent and symptomatic and could not be resolved using conservative measures. Any symptoms caused by the edema were not defined as independent complications but, rather, as secondary to the underlying edema and therefore were not reflected in the overall complication rate. The evaluation of all MR images (preoperative and postoperative) involved a neuroradiologist. For patients with a tumor, radiographic response was assessed immediately after surgery, 24 hours after surgery, and then at the first follow-up visit. For patients with radiation necrosis/recurrent metastasis, only a small subset underwent advanced MRI (MRI spectroscopy), and distinction was difficult in all cases, even in the small number of patients who underwent biopsy. All 37 of these patients exhibited in-field recurrence, and the decision to intervene was made on the basis of radiological progression over several scans.

The Student t-test was used for statistical analysis of operative parameters and duration of intensive care unit (ICU) and hospital stays among different indications. Similarly, a comparison was made between the initial 20 cases and the following 82 cases. We identified the initial 20 cases as part of our learning curve, because the experience from these initial cases resulted in a protocol that became standard for the remaining 82 patients. A new symptom or deficit was considered a complication if it was a direct result of the procedure. Readmission rates were calculated using the standard method according to America's Health Insurance Plans Center for Policy and Research guidelines. ${ }^{5}$ Medical records were reviewed to identify any readmissions that the patient had to the same hospital within 30 days of discharge from the procedure. Emergency department visits, readmissions for scheduled second MRgLITT procedures, and unrelated readmissions were not included in those values. A readmission was considered related if the reason or complication that required readmission was secondary to or exacerbated by the initial procedure. That number of readmissions was then divided by the total number of admissions for our 102 patients (including the readmissions) to result in the readmission rate for our center.

\section{Results}

Operative parameters are shown in Table 2. The total average operative time $( \pm \mathrm{SD})$ for all patients at our center was $170.5 \pm 34.4$ minutes $(2.8 \pm 0.6$ hours $) ; 43.6 \pm 11.8$ minutes were spent for preparation (intraoperative preincision steps), $34.2 \pm 10.0$ minutes in the operating room, $42.8 \pm 12.0$ minutes for transfer for MRI, and $47.3 \pm 20.6$ minutes in the MRI suite, and the average ablation time was $8.7 \pm 6.8$ minutes $(521.8 \pm 407.2$ seconds). Stratified operative parameters based on patient diagnoses are also shown in Table 2. A comparison of operative parameters between the initial 20 and remaining 82 patients revealed a significant difference in laser $(\mathrm{p}=0.0187)$ and total operative $(\mathrm{p}=0.0044)$ times.

\section{Neurological Complications}

The most common complication was the development of new neurological deficits (14 cases). The most prevalent neurological deficit was motor deficit $(\mathrm{n}=12)$, including facial droop, paresis, hemiplegia, and gait instability. Other neurological deficits included unexpected cognitive decline $(\mathrm{n}=1)$ and vision problems such as diplopia $(\mathrm{n}=1)$. Patients were followed up by the senior author (S.F.D.) during the postoperative period. Tables 3 and 4 summarize perioperative complications in this series. As shown in Table 5, of the 14 patients who showed neurological symptoms, 64.3\% (9 of 14) had complete resolution of deficits within 1 month, $7.1 \%$ ( 1 of 14) had partial resolution of deficits within 1 month, $14.3 \%$ (2 of 14) had not had resolution of deficits at the most recent follow-up, and $14.3 \%$ ( 2 of 14) died as a result of progression without resolution of deficits.

TABLE 2. Operative parameters*

\begin{tabular}{ccccccc}
\hline Parameter & Prep Time $(\mathrm{mins})$ & OR Time $(\mathrm{mins})$ & Transfer Time $(\mathrm{mins})$ & MRI Time (mins) & Laser Time (mins) & Total Time (hrs) \\
\hline Diagnosis & & & & & & \\
\hline Primary tumor & $40.5 \pm 10.2(40)$ & $34.0 \pm 10.8(40)$ & $43.9 \pm 13.9(40)$ & $50.6 \pm 19.9(40)$ & $9.3 \pm 6.5(50)$ & $2.9 \pm 0.6(44)$ \\
\hline RecMets/RadNec & $47.4 \pm 13.5(27)$ & $32.8 \pm 9.3(27)$ & $42.6 \pm 9.8(27)$ & $47.0 \pm 24.1(27)$ & $8.7 \pm 8.1(36)$ & $2.8 \pm 0.6(28)$ \\
\hline Pain & $46.3 \pm 6.3(4)$ & $41.3 \pm 7.5(4)$ & $40.0 \pm 7.1(4)$ & $37.5 \pm 9.6(4)$ & $4.3 \pm 0.6(5)$ & $2.9 \pm 0.3(4)$ \\
\hline Epilepsy & $44.4 \pm 12.6(9)$ & $36.1 \pm 9.3(9)$ & $39.4 \pm 11.3(9)$ & $37.8 \pm 10.9(9)$ & $7.6 \pm 2.3(9)$ & $2.6 \pm 0.4(9)$ \\
\hline Initial 20 cases & $44.0 \pm 10.8(5)$ & $45.0 \pm 15.0(5)$ & $42.0 \pm 16.4(5)$ & $84.0 \pm 32.9(5)$ & $14.0 \pm 11.3(20)$ & $3.7 \pm 0.7(8)$ \\
\hline Later 82 cases & $43.5 \pm 12.0(75)$ & $33.5 \pm 9.3(75)$ & $42.8 \pm 11.8(75)$ & $44.9 \pm 17.2(75)$ & $7.4 \pm 4.3(80)$ & $2.8 \pm 0.5(77)$ \\
\hline p Value & 0.9301 & 0.1613 & 0.9196 & 0.0556 & $0.0187 \dagger$ & $0.0044 \dagger$ \\
\hline Average & $43.6 \pm 11.8(80)$ & $34.2 \pm 10.0(80)$ & $42.8 \pm 12.0(80)$ & $47.3 \pm 20.6(80)$ & $8.7 \pm 6.8(100)$ & $2.8 \pm 0.6(85)$ \\
\hline
\end{tabular}

$\mathrm{OR}=$ operating room.

* Values are presented as the mean \pm SD (values/numbers of cases for which this information was available).

$\dagger$ Significant result (two-tailed t-test analysis). 
TABLE 3. Complications

\begin{tabular}{cl}
\hline \multicolumn{1}{c}{ Complication } & No. $(\%)^{*}$ \\
\hline Neurological deficits & $14(13.7)$ \\
\hline Motor deficits & 12 \\
\hline Cognitive decline & 1 \\
\hline Vision \& eye problems & 1 \\
\hline Hemorrhage & $3(2.9)$ \\
\hline Insertional hemorrhage & 2 \\
\hline Ablation-induced hemorrhage & 1 \\
\hline Refractory edema & $5(4.9)$ \\
\hline Infection & $2(2.0)$ \\
\hline Inaccurate laser placement & $2(2.0)$ \\
\hline Thermal injury & $1(1.0)$ \\
\hline Total & $27(26.5)$ \\
\hline
\end{tabular}

* Percentages are relative to all cases in the study $(n=102)$.

Of the 14 patients in whom new neurological deficit developed, a majority had either partial or full resolution of their deficits by 1 month. In examining the postoperative MRI sequences of these cases, motor deficits correlated with the proximity to the corticospinal tracts. As the local effects resolved, there was a resolution of the corresponding neurological deficit as well. For the 10 patients in whom the deficits resolved, we concluded that postoperative edema in the ablation bed was the cause. For the 2 patients without resolution, a unilateral upper-extremity motor deficit was noted. Although no specific additional MRI changes were identified, we suspect that this deficit occurred as a result of permanent thermal injury to the motor tract. For the 2 patients who died, 1 lesion was relatively large and the other was within the midbrain and pons. Rapid development of malignant edema in both of these patients led to global neurological decline.

\section{Procedural Complications}

Perioperative complications included insertional and
TABLE 5. Resolution of neurological deficits

\begin{tabular}{lc}
\hline \multicolumn{1}{c}{ Outcome } & No. of Patients $(\%)^{*}$ \\
\hline Complete resolution of deficits w/in 1 mo & $9(64.3)$ \\
\hline Partial resolution of deficits w/in 1 mo & $1(7.1)$ \\
\hline No resolution of deficits at most recent follow-up & $2(14.3)$ \\
\hline Patient died w/o resolution of deficits & $2(14.3)$ \\
\hline Total no. of patients w/ neurological deficits & $14(100)$ \\
\hline
\end{tabular}

* Percentages are relative to the number of cases with neurological deficits, not the total number of cases in our study.

ablation-induced hemorrhages that required craniotomy and evacuation $(\mathrm{n}=3)$, laser misplacement that led to procedure abortion $(n=2)$, postprocedural refractory edema $(n=5)$, and infection at the incisional site $(n=2)$. Figure 1 shows an insertional hemorrhage in 1 of our patients. Of the 3 patients with insertional hemorrhage, 2 did not require open surgery as an intervention until after MRgLITT completion. There was 1 case of thermal injury to the pituitary gland that led to secondary complications. Two perioperative deaths occurred, and a third death occurred within the same hospital stay as the MRgLITT but was not attributable to the procedure itself.

The procedure could not be completed in 2 patients. An 85-year-old with recurrent meningioma developed an insertional hemorrhage while undergoing MRgLITT. This hemorrhage required emergent evacuation through a craniotomy. Secondary to this complication, the patient suffered permanent cognitive dysfunction. In the second case, a 65-year-old man with hemangioblastoma had an inaccurately placed laser that necessitated abortion of the procedure. The patient ultimately underwent open resection and did well.

The 2 perioperative deaths occurred secondary to refractory edema after the MRgLITT procedure. The first case was of an 84-year-old man with glioblastoma multiforme who underwent ablation of a $60-\mathrm{cm}^{3}$ mass. Postoperative imaging revealed malignant intracranial edema that required hemicraniotomy. After the emergency pro-

TABLE 4. Complications according to diagnosis and the initial 20 versus 82 remaining patients

\begin{tabular}{|c|c|c|c|c|c|c|c|}
\hline Complication & Neuro Deficits & Hemorrhage & Edema & Infection & $\begin{array}{c}\text { Inaccurate Laser } \\
\text { Placement }\end{array}$ & $\begin{array}{l}\text { Thermal } \\
\text { Injury }\end{array}$ & Death \\
\hline \multicolumn{8}{|l|}{ According to diagnosis (no.) } \\
\hline Primary brain tumor & 7 & 2 & 4 & 1 & 2 & 0 & 2 \\
\hline RecMets/RadNec & 7 & 1 & 1 & 1 & 0 & 1 & 0 \\
\hline Pain & 0 & 0 & 0 & 0 & 0 & 0 & 0 \\
\hline Epilepsy & 0 & 0 & 0 & 0 & 0 & 0 & 0 \\
\hline Total & 14 & 3 & 5 & 2 & 2 & 1 & 2 \\
\hline \multicolumn{8}{|c|}{ According to initial 20 vs remaining 82 patients } \\
\hline Initial 20 patients (no.) & 1 & 1 & 1 & 0 & 2 & 1 & 1 \\
\hline Complication rate $(\%)^{*}$ & 5 & 5 & 5 & 0 & 10 & 5 & 5 \\
\hline Remaining 82 patients (no.) & 13 & 2 & 4 & 2 & 0 & 0 & 1 \\
\hline Complication rate $(\%)$ & 16 & 2 & 5 & 2 & 0 & 0 & 1 \\
\hline Total (no.) & 14 & 3 & 5 & 2 & 2 & 1 & 2 \\
\hline
\end{tabular}

Neuro $=$ neurological.

* Complication rate is per individual group of patients, not total number of patients in the study. 

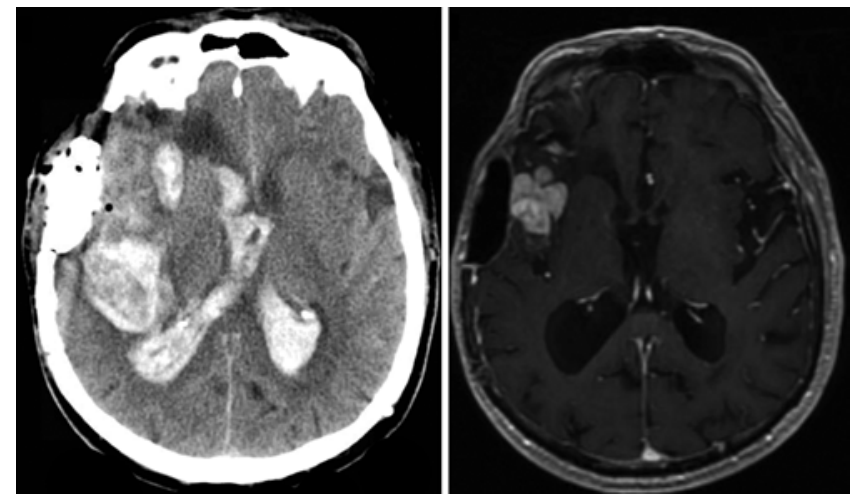

FIG. 1. Insertional hemorrhage. Left: Postoperative CT image showing a hemorrhage that resulted after insertion of the laser catheter. Right: Preoperative MR image showing the recurrent meningioma for which the patient underwent MRgLITT.

cedure, the patient failed to regain consciousness and died during the same admission. The second case involved a 57-year-old man with a history significant of glioblastoma of the midbrains and pons. Postoperatively, the patient developed edema secondary to the procedure that required a medically induced coma and led to an extended stay in the ICU. The patient was discharged to a long-term care center, and he remained comatose until death as a result of progression of the disease. There was a third death in our series that, although not secondary to the procedure, occurred as a result of the patient's underlying conditions and progression of disease. The patient was a 15-monthold boy with teratoma in the third ventricle. Although the procedure was uneventful, the patient developed rapid progression of disease. As seen with follow-up imaging, the tumor had extended through the subarachnoid space and circumferentially involved the brainstem and most parts of the spinal cord. The patient was unable to support respiration and died as a result. An argument could certainly be made that the procedure should never have been done, but that discussion is beyond the scope of this article.

Table 4 further stratifies complications and perioperative deaths based on diagnosis and compares our initial 20 patients versus the remaining 82 patients. All the complications and deaths occurred in patients with intracranial tumors. There were no complications in patients who underwent the procedure for chronic pain syndrome or epilepsy. Table 4 also breaks down the rates of occurrence within our 2 groups (initial 20 patients and remaining 82 patients).

\section{Radiological Observations}

Radiological responses to MRgLITT were similar to what we have reported in previous studies. ${ }^{19}$ The most frequent postoperative imaging schedule was immediately after surgery, 24 hours after surgery, and at first followup (typically 1 month). Although specific volumetric assessments and detailed analysis are beyond the scope of this paper, there was an overall increase in apparent lesion size immediately after surgery and reduction by 24 hours after surgery. Sizes 24 hours after surgery were similar to those at first follow-up. In patients with recurrent metastasis/radiation necrosis, edema was reduced with time; however, in 1 patient, edema increased, and we have listed it as a complication. We suspect that this example in which edema increased was likely a result of incomplete ablation and residual lesion.

\section{Hospital Stay and Readmissions}

The average ( \pm SD) ICU stay was $1.8 \pm 3.4$ days, and the average total inpatient stay was $3.6 \pm 5.4$ days. The median duration of stay for both the ICU and the hospital was 1 day. We also further categorized our patients as part of either the initial 20 patients, whom we identified as our learning curve, and the remaining 82 patients. The times spent in the ICU and hospital for our initial patients were $3.5 \pm 6.2$ days and $5.2 \pm 8.5$ days, respectively. For the remaining patients, the times spent in the ICU and hospital were $1.4 \pm 2.2$ and $3.2 \pm 4.4$ days, respectively. Although the average lengths of the ICU and hospital stays were lower in the later 82 cases, this difference was not statistically significant. Table 6 summarizes these results. Of all the patients, disposition data were available for 84 patients; $59.5 \%(\mathrm{n}=50)$ of the patients were discharged to home, $38.1 \%(\mathrm{n}=32)$ were discharged to a rehabilitation facility, and $2.4 \%(\mathrm{n}=2)$ died in the hospital before discharge.

There were 6 readmissions within 30 days of discharge after the procedure. The readmission rate for our center was $5.6 \%$, which does not include any readmissions that were preplanned for a scheduled procedure or visits to the emergency department in which the patient was treated in the emergency department and discharged home. There were 2 readmissions for scheduled procedures and 2 emergency department visits (without inpatient admission) within 30 days after discharge that were not included in the readmission rate.

\section{Discussion}

MRgLITT is a useful, minimally invasive method for treating a wide range of intracranial pathologies. We present here our experience with 102 patients seen at our institution, along with their operative parameters and com-

TABLE 6. ICU and hospital stays*

\begin{tabular}{cl}
\hline \multicolumn{1}{c}{ Inpatient Stay } & LOS (days) $\dagger$ \\
\hline ICU & \\
\hline Initial 20 patients & $3.5 \pm 6.2$ \\
\hline Remaining 82 patients & $1.4 \pm 2.2$ \\
\hline Overall average & $1.8 \pm 3.4$ \\
\hline Median & 1.0 \\
\hline Hospital $\neq$ & \\
\hline Initial 20 patients & $5.2 \pm 8.5$ \\
\hline Remaining 82 patients & $3.2 \pm 4.4$ \\
\hline Overall average & $3.6 \pm 5.4$ \\
\hline Median & 1.0 \\
\hline
\end{tabular}

LOS = length of stay.

* Nonsignificant t-test analysis.

† Values are presented as the mean \pm SD unless otherwise stated.

$\ddagger$ Days spent in hospital as inpatient after MRgLITT; excludes any days of

hospitalization before the procedure. 
plications. The purpose of our study was to report our experience and emphasize the use of MRgLITT as a safe additional treatment modality for treating intracranial lesions, epilepsy, and pain.

Although MRgLITT is becoming part of the neurosurgical armamentarium, the benefits and risks of the approach are important to consider. Aside from the minimal invasiveness of the approach, real-time monitoring of both thermal distribution and damage allow for a high degree of therapy control. Furthermore, the approach typically involves a shorter or no ICU stay, a reduced overall length of hospital stay, and, theoretically, a lower risk of common hospital complications (pneumonia, deep venous thrombosis, and urinary tract infection). The risks of MRgLITT can be placed in 2 key categories: 1) related to laser insertion and 2) related to thermal ablation. Risks related to laser insertion are similar to those of biopsy, insertional hemorrhage after a depth electrode procedure, and misplacement. Related to the ablation are subtotal ablation and unintended thermal damage, both of which are important when deciding the role of MRgLITT in a particular case. Lesions with complex geometry and those near critical structures may be problematic. The efficacy of MRgLITT is beyond the scope of this paper; however, we have reported our outcomes in several previous studies. . $18,18^{18}$ Our series included primary brain tumors, metastasis/radiation necrosis, epilepsy, and chronic pain syndromes (cingulotomy). MRgLITT was chosen over other methods of treatment when 1) other treatments, including traditional resection, radiation, and chemotherapy, were attempted and exhausted, 2) the lesion was deemed inoperable through traditional surgical means, and/or 3) the patient preferred MRgLITT over other methods.

Operative parameters were compared between our initial group of 20 patients and the following 82 patients. A distinction was made between the first 20 patients and subsequent patients because experience in the former was part of our learning curve and led to the development of a standardized MRgLITT procedure protocol at our institution. Compared with our initial patients, subsequent patients required a significant decrease in the laser ablation and total operative times. We found that, with experience, we were able to better position the laser catheters to optimize the delivery of thermal therapy while reducing laser-on time.

Despite the medically compromised and debilitating states of our patients, the occurrence of severe complications and neurological deficits was relatively low, and the majority of these deficits fully resolved. The majority of the complications encountered were new neurological deficits $(n=14)$, of which, $64.3 \%(n=9)$ had complete resolution of all deficits, $7.1 \%(1 \mathrm{n}=)$ had partial resolution, and $14.3 \%(\mathrm{n}=2)$ died before or without resolution of symptoms secondary to their disease. The 2 patients without resolution did not have any specific findings on MRI that would correlate to their deficit. However, both patients had white matter within the vicinity of the ablation bed. We suspect that there may have been mild thermal injury to these areas that resulted in damage. To date, their deficits have had minimal resolution.

More serious surgical complications included refractory edema, hemorrhage, and perioperative death. Refrac- tory edema was seen in 5 patients with a tumor, and there were 2 deaths. One of these patients had a very large mass and, thus, the risk for life-threatening edema was high. The other patient had a mass in the midbrain and pons and also was at high risk for edema-induced injury. Hemorrhage was seen in 3 patients, all of whom required open surgery at some point in the postoperative time frame to evacuate the hemorrhage. Hemorrhage is a risk with any procedure involving intracranial insertion of a catheter. In these 3 patients, despite our careful trajectory planning, vessels along the catheter tract were violated. For some of these patients, the argument could certainly be made that the procedure should never have been done, but that discussion is beyond the scope of this article. Nonetheless, these examples provide evidence that, although MRgLITT is less invasive, there are real risks that may lead to serious complications. Because of the complications associated with edema, we have generally stopped offering this procedure to patients who require high preoperative steroid doses for symptom relief. In addition, we have become more aggressive with surveillance and often treat before the development of symptomatic edema to prevent its occurrence postoperatively.

All of the complications and perioperative deaths were seen strictly in patients who underwent the MRgLITT procedure for primary or metastatic intracranial tumors. The patients in our series with chronic pain syndrome or epilepsy as the indication for the procedure experienced no complications or death. The higher number of complications within the population of patients with a tumor may be a result of the significantly higher number of patients with a tumor than patients with pain or epilepsy. Patients with primary or metastatic tumor pathologies may also be in a more compromised state of health before undergoing the procedure, which puts them at greater risk for the development of complications. Most patients with a tumor underwent this procedure after all other options had been exhausted and so were at the end of their disease overall. In addition, when we compared our initial experience with the procedure with that in later cases, we found the incidence of direct or major procedural complications to be higher or equal in the first 20 patients. The incidence of hemorrhage, inaccurate laser placement, thermal injury, and perioperative death decreased with time and experience. However, the percentage of neurological deficits increased from $5 \%$ to $16 \%$. It is likely that the initial $5 \%$ rate was not truly representative because the initial group had only 20 subjects. In retrospect, we were more conservative with our degree of ablation and chose less complex cases-almost all these lesions were within the confines of a first-order ellipse. As we developed more experience, we applied MRgLITT to more complex lesions. The majority of deficits resolved within 1 month. MRgLITT is not applicable in all cases, especially those with highly multilobar irregular morphology or in highly eloquent brain areas. Overall, we believe that MRgLITT should be seen as a potential treatment option, not necessarily a replacement for craniotomy.

Although not statistically significant, the duration of stay in the ICU and on the regular floor after MRgLITT followed a decreasing trend after the initial 20 patients 
as our learning curve was overcome. This observation of relatively short hospital and ICU stays was consistent with those reported by other authors. A prospective study by Hawasli et al. ${ }^{9}$ of 17 patients who underwent MRgLITT found that the average hospital stay was $5.0 \pm 6.4$ days and the average ICU time was $1.8 \pm 1.7$ days. In cases that involve a low risk for postoperative symptomatic edema, no intraoperative complications, and good postanesthesia status, we often have the patients go to a step-down unit to avoid ICU stay completely. In an earlier clinical trial by Schwarzmaier et al., ${ }^{27}$ who used MRgLITT for treatment of glioblastomas, none of the 16 patients required a stay in the ICU after surgery. Of 7 patients who underwent MRgLITT for metastatic tumors in a study by Carpentier et al., ${ }^{3}$ nearly all of the patients were discharged within 24 hours, and the average hospital stay was 26 hours.

When compared with more traditional alternatives for corresponding pathologies, MRgLITT is associated with a shorter hospital stay. In 1998, Sawaya et al. ${ }^{25}$ presented a series of 400 craniotomies for treating parenchymal tumors and reported a median postoperative hospital stay of 5 days. Long et al. ${ }^{12}$ reviewed 4723 craniotomies done for tumors and found the average hospital stay to be 6.8 days at high-volume hospitals and 8.8 days in low-volume hospitals. Additional studies with more standard craniotomies for tumor resection also reflected a similar trend in mean length of hospital stay. ${ }^{17,30}$ Although the impact of a shorter hospital stay on the risk of common postoperative morbidities (i.e., pneumonia, urinary tract infection, deep venous thrombosis) is clear, we could not determine from our study whether this risk is reduced compared to that with traditional surgical approaches.

The readmission rate at our center among 102 patients was $5.6 \%$. Four patients had a single readmission each, and 1 patient had 2 readmissions within the 30 days. The readmission diagnoses included cerebral edema $(n=2)$, altered mental status $(n=2)$, and seizures $(n=2$; both of these patients had a history of seizure disorder). Guidelines from the America's Health Insurance Plans Center for Policy and Research were used to calculate our readmission rates. ${ }^{5}$ This method was chosen over other calculation methods because of its wide use among studies and its simplicity. In a study by Marcus et al., ${ }^{13}$ the 30day readmission rate for patients in whom craniotomy was performed to treat malignant supratentorial tumors over a 15 -year span was $13.2 \%$. In their study, of a total of 18,506 patients, 1790 had at least 1 readmission within 30 days of discharge. Our study was much smaller, and thus our results cannot in any way directly compare with those of more invasive procedures. However, there may be utility in larger studies to examine readmission rates in the context of MRgLITT and other options.

Overall, as is true for any other neurosurgical procedure, complications can be minimized with strict attention to technique and respect for the pathology and its location. One of the issues with the procedure itself is that it does not enable visualization of critical pathways during the ablation process. The ability to overlay critical fiber tracts onto the reference image during ablation may significantly reduce this risk. Another option is to perform the procedure with the patient awake. We found this option to be exceedingly difficult, because any movement during ablation forces the procedure to restart. We hope that future advances in thermal monitoring will reduce the risk associated with ablation near critical functional structures.

As with many retrospective single-institution case series, our study has several limitations. Our sample size was still too small to draw any definitive conclusions. The retrospective nature introduced limitations in case selection, uniformity of collected data, and recall bias. In addition, we had a diverse collection of diagnoses, which further decreases the power of a single diagnosis and its safety profile with this procedure. Individual diagnoses and size and location of the tumor, among other factors, should be considered strongly on an individual basis before choosing MRgLITT as a treatment option. A key controversial component of our work will be our lack of distinction between cases of radiation necrosis and those of recurrent metastasis. Although these are 2 potentially different processes, radiographic distinction is very difficult, even with advanced imaging techniques. The presumed gold standard is biopsy; however, even biopsy is fraught with sampling bias and a lack of concordance between pathologists. ${ }^{24,31}$ Among our 37 patients whom we grouped as having radiation necrosis/recurrent metastasis, all had received previous maximal radiation. Among our patients, a small but important number developed significant complications. The degree of ablation, composition of surrounding structures, and location of ablation all may likely predict potential for significant edema and/or neurological deficits. These factors would need to be studied in more detail, and we hope that our study can trigger future studies.

\section{Conclusions}

MRgLITT is promising as an efficient and effective treatment option for a wide range of neurological pathologies. In our study, this technique resulted in relatively few complications, many of which were temporary neurological deficits that were significantly improved with therapy. Although MRgLITT is gaining momentum in the neurosurgical community, large prospective studies are yet to be undertaken. Nonetheless, we hope that our results can be of use to others using MRgLITT for intracranial pathologies.

\section{References}

1. Brace CL, Hinshaw JL, Lubner MG: Thermal ablation for the treatment of abdominal tumors. J Vis Exp (49):2596, 2011

2. Carpentier A, Chauvet D, Reina V, Beccaria K, Leclerq D, McNichols RJ, et al: MR-guided laser-induced thermal therapy (LITT) for recurrent glioblastomas. Lasers Surg Med 44:361-368, 2012

3. Carpentier A, McNichols RJ, Stafford RJ, Guichard JP, Reizine D, Delaloge S, et al: Laser thermal therapy: real-time MRI-guided and computer-controlled procedures for metastatic brain tumors. Lasers Surg Med 43:943-950, 2011

4. Carpentier A, McNichols RJ, Stafford RJ, Itzcovitz J, Guichard JP, Reizine D, et al: Real-time magnetic resonance-guided laser thermal therapy for focal metastatic brain tumors. Neurosurgery 63 (1 Suppl 1):ONS21-ONS29, 2008

5. Center for Policy and Reseach: Simple Methods of Measuring Hospital Readmission Rates. Washington, DC: America's Health Insurance Plans, 2012

6. Curry DJ, Gowda A, McNichols RJ, Wilfong AA: MR-guid- 
ed stereotactic laser ablation of epileptogenic foci in children. Epilepsy Behav 24:408-414, 2012

7. Esquenazi Y, Kalamangalam GP, Slater JD, Knowlton RC, Friedman E, Morris SA, et al: Stereotactic laser ablation of epileptogenic periventricular nodular heterotopia. Epilepsy Res 108:547-554, 2014

8. Harsh V, Viswanathan A: Surgical/radiological interventions for cancer pain. Curr Pain Headache Rep 17:331, 2013

9. Hawasli AH, Bagade S, Shimony JS, Miller-Thomas M, Leuthardt EC: Magnetic resonance imaging-guided focused laser interstitial thermal therapy for intracranial lesions: singleinstitution series. Neurosurgery 73:1007-1017, 2013

10. Jethwa PR, Barrese JC, Gowda A, Shetty A, Danish SF: Magnetic resonance thermometry-guided laser-induced thermal therapy for intracranial neoplasms: initial experience. Neurosurgery 71 (1 Suppl Operative):133-145, 2012

11. Kahn T, Harth T, Kiwit JC, Schwarzmaier HJ, Wald C, Mödder U: In vivo MRI thermometry using a phase-sensitive sequence: preliminary experience during MRI-guided laserinduced interstitial thermotherapy of brain tumors. J Magn Reson Imaging 8:160-164, 1998

12. Long DM, Gordon T, Bowman H, Etzel A, Burleyson G, Betchen S, et al: Outcome and cost of craniotomy performed to treat tumors in regional academic referral centers. Neurosurgery 52:1056-1065, 2003

13. Marcus LP, McCutcheon BA, Noorbakhsh A, Parina RP, Gonda DD, Chen C, et al: Incidence and predictors of 30-day readmission for patients discharged home after craniotomy for malignant supratentorial tumors in California (19952010). J Neurosurg 120:1201-1211, 2014

14. McNichols RJ, Gowda A, Kangasniemi M, Bankson JA, Price RE, Hazle JD: MR thermometry-based feedback control of laser interstitial thermal therapy at $980 \mathrm{~nm}$. Lasers Surg Med 34:48-55, 2004

15. McNichols RJ, Kangasniemi M, Gowda A, Bankson JA, Price RE, Hazle JD: Technical developments for cerebral thermal treatment: water-cooled diffusing laser fibre tips and temperature-sensitive MRI using intersecting image planes. Int J Hyperthermia 20:45-56, 2004

16. Norred SE, Johnson JA: Magnetic resonance-guided laser induced thermal therapy for glioblastoma multiforme: a review. BioMed Res Int 2014:761312, 2014

17. Paleologos TS, Wadley JP, Kitchen ND, Thomas DG: Clinical utility and cost-effectiveness of interactive image-guided craniotomy: clinical comparison between conventional and image-guided meningioma surgery. Neurosurgery 47:4048, 2000

18. Patel NV, Agrawal N, Mammis A, Danish SF: Frameless stereotactic MRI-guided laser interstitial thermal therapy to perform anterior cingulotomy for intractable pain: feasibility, technical aspects, and initial experience in three patients. Neurosurgery 11 (Suppl 2):17-25, 2015

19. Patel NV, Jethwa PR, Barrese JC, Hargreaves EL, Danish SF: Volumetric trends associated with MRI-guided laser-induced thermal therapy (LITT) for intracranial tumors. Lasers Surg Med 45:362-369, 2013

20. Postma EL, van Hillegersberg R, Daniel BL, Merckel LG, Verkooijen HM, van den Bosch MA: MRI-guided ablation of breast cancer: where do we stand today? J Magn Reson Imaging 34:254-261, 2011

21. Rahmathulla G, Recinos PF, Kamian K, Mohammadi AM, Ahluwalia MS, Barnett GH: MRI-guided laser interstitial thermal therapy in neuro-oncology: a review of its current clinical applications. Oncology 87:67-82, 2014

22. Rahmathulla G, Recinos PF, Valerio JE, Chao S, Barnett GH: Laser interstitial thermal therapy for focal cerebral radiation necrosis: a case report and literature review. Stereotact Funct Neurosurg 90:192-200, 2012

23. Raimbault A, Cazals X, Lauvin MA, Destrieux C, Chapet S,
Cottier JP: Radionecrosis of malignant glioma and cerebral metastasis: a diagnostic challenge in MRI. Diagn Interv Imaging 95:985-1000, 2014

24. Rao MS, Hargreaves EL, Khan AJ, Haffty BG, Danish SF: Magnetic resonance-guided laser ablation improves local control for postradiosurgery recurrence and/or radiation necrosis. Neurosurgery 74:658-667, 2014

25. Sawaya R, Hammoud M, Schoppa D, Hess KR, Wu SZ, Shi WM, et al: Neurosurgical outcomes in a modern series of 400 craniotomies for treatment of parenchymal tumors. Neurosurgery 42:1044-1056, 1998

26. Schulze PC, Kahn T, Harth T, Schwurzmaier HJ, Schober $\mathrm{R}$ : Correlation of neuropathologic findings and phase-based MRI temperature maps in experimental laser-induced interstitial thermotherapy. J Magn Reson Imaging 8:115-120, 1998 (Erratum in J Magn Reson Imaging 14:658, 2001)

27. Schwarzmaier HJ, Eickmeyer F, von Tempelhoff W, Fiedler VU, Niehoff H, Ulrich SD, et al: MR-guided laser-induced interstitial thermotherapy of recurrent glioblastoma multiforme: preliminary results in 16 patients. Eur J Radiol 59:208-215, 2006

28. Sommer G, Bouley D, Gill H, Daniel B, Pauly KB, Diederich C: Focal ablation of prostate cancer: four roles for magnetic resonance imaging guidance. Can J Urol 20:6672-6681, 2013

29. Streitparth F, Teichgräber U, Walter T, Schaser KD, Gebauer B: Recurrent osteoid osteoma: interstitial laser ablation under magnetic resonance imaging guidance. Skeletal Radiol 39:1131-1137, 2010

30. Tan TC, Black PM: Image-guided craniotomy for cerebral metastases: techniques and outcomes. Neurosurgery 61 (1 Suppl):349-357, 2007

31. Tiwari P, Danish S, Madabhushi A: Identifying MRI markers to evaluate early treatment related changes post laser ablation for cancer pain management. Proc SPIE Int Soc Opt Eng 9036: $90362 \mathrm{~L}, 2014$

32. van den Bosch M, Daniel B, Rieke V, Butts-Pauly K, Kermit E, Jeffrey S: MRI-guided radiofrequency ablation of breast cancer: preliminary clinical experience. J Magn Reson Imaging 27:204-208, 2008

33. Vogl TJ, Naguib NN, Lehnert T, Nour-Eldin NE: Radiofrequency, microwave and laser ablation of pulmonary neoplasms: clinical studies and technical considerations-review article. Eur J Radiol 77:346-357, 2011

34. Vogl TJ, Straub R, Zangos S, Mack MG, Eichler K: MRguided laser-induced thermotherapy (LITT) of liver tumours: experimental and clinical data. Int J Hyperthermia 20:713724,2004

35. Yarmolenko PS, Moon EJ, Landon C, Manzoor A, Hochman DW, Viglianti BL, et al: Thresholds for thermal damage to normal tissues: an update. Int J Hyperthermia 27:320-343, 2011

\section{Disclosures}

Dr. Danish has received honoraria from Medtronic, Inc.

\section{Author Contributions}

Conception and design: all authors. Acquisition of data: P Patel. Analysis and interpretation of data: P Patel. Drafting the article: P Patel. Critically revising the article: all authors. Reviewed submitted version of manuscript: all authors. Approved the final version of the manuscript on behalf of all authors: NV Patel. Statistical analysis: P Patel. Administrative/technical/material support: Danish. Study supervision: NV Patel, Danish.

\section{Correspondence}

Nitesh V. Patel, Division of Neurosurgery, Rutgers-Robert Wood Johnson Medical School, 125 Paterson St., CAB Suite 2100, New Brunswick, NJ 08901. email: patel236@njms.rutgers.edu. 